\title{
Perception of earbuds side effects among teenager and adults in Eastern Province of Saudi Arabia: A cross sectional study
}

\author{
Abdullah A. Alarfaj ${ }^{\text {a, }}$, Lujain M. AlAhmmed ${ }^{\mathrm{b}}$, Sayed Ibrahim Ali ${ }^{\mathrm{c}}$ \\ ${ }^{a}$ Department of Otolaryngology and Head and Neck Surgery, College of Medicine, King Faisal University, Alahsa, Saudi Arabia \\ ${ }^{\mathrm{b}}$ College of Medicine, King Faisal University, Alahsa, Saudi Arabia \\ ${ }^{c}$ Department of Biostatistics, College of Medicine, King Faisal University, Alahsa, Saudi Arabia
}

A R T I C L E I N F O

\section{Keywords:}

Earbuds

Headphones

Side effects

Awareness

Saudi Arabia

\begin{abstract}
A B S T R A C T
Background: As technology keeps on developing, earbuds have been more popular recently among different age groups. As they are inserted into the external ear canal, they obstruct and isolate its communication with the outer environment. Thus, changing temperature and humidity inside the external ear canal. (EEC), and the extent of those environmental changes is influenced by the duration of using the earbuds, as well as, the attitude of maintaining hygiene of the earpieces.

Material and methods: A cross sectional study was conducted among the teenager and adult population of AlAhssa, Dammam, AlQatif, and Jubail Industrial cities, Eastern Province, Saudi Arabia. An online validated voluntary self-administered questionnaire was disseminated. The questionnaire consisted of 19 elements including consent. The net data of 504 participants was analyzed with SPSS version 21 .

Results: Regular use of earbuds was found among $32.5 \%$ of the respondents. The mean knowledge score was 2.49 (SD 0.95) out of 4 points. Good, moderate and poor awareness were found among 49.2\%, 35.3\% and 15.5\% respectively. The most commonly known symptoms of prolonged use of earbuds were itching (28\%), otalgia $(23 \%)$ and excessive ear wax $(21.8 \%)$. It was found that females $(\mathrm{T}=-2.569 ; \mathrm{P}=0.010)$ and earbuds users for $1-2 \mathrm{~h}$ per day $(\mathrm{F}=2.901 ; \mathrm{P}=0.040)$ have significantly better awareness while students $(\mathrm{F}=2.824 ; \mathrm{P}=0.044)$ and those using high volume level have significantly poorer level of awareness.

Conclusion: There was an adequate awareness toward the side effects of excessive usage of earbuds among teenagers and young adults. Females showed better awareness compared to males and short-term usage of earbuds was a significant predictor of good awareness.
\end{abstract}

\section{Introduction}

The 1880's is the age hearing devices (basically headphones) were traced to. They were only used by telephone operators. ${ }^{1,2}$ In 1910 , the Navy of the United States of America incorporated the use of headphones manufactured by Nathaniel Baldwin. ${ }^{2,3}$ In 1937, the first dynamic headphones were introduces into the market. ${ }^{2,4} 1979$ was the beginning of the portable era. ${ }^{2,5}$ It was mainly because the Walkman was first introduced by Sony. ${ }^{2,5}$ In year 1980's, the ear-phones/buds started gaining popularity. ${ }^{2,6}$ Nevertheless, they did not peak in popularity until the year of 2001, when Apple invented the iPod Earbuds/Earphones are cheaper, lighter and smaller in comparison to all types of headphones. ${ }^{7}$ Earbuds/earphones are designed and manufactured to be inserted into the external ear canal. ${ }^{7}$ Earbuds combine microphone's and headphones' characteristics. ${ }^{7}$

This study aims toward assessing the awareness among the population of Alahssa, Dammam, Alqatif, and Jubail Industrial Cities in regard of the direct relationship between less frequent use and less side effects of earbuds, the immediate side effects of extensive use of earbuds (headache, tinnitus, otalgia, excessive ear wax, itching.), the long-term side effects of extensive use of earbuds (hearing loss, external ear infections including Otitis Externa or Folliculitis.), and to compare between males' and females' level of awareness. Also, to reflect the common hygienic habits related to earbuds-use among the general population like estimating population tendency to clean their earbuds and to exchange earbuds. Finally, Compare the percentage of earbuds users and headphones users.

\footnotetext{
* Corresponding author. Department of Otolaryngology and Head and Neck surgery, King Faisal University, Alahsa, Saudi Arabia.

E-mail address: aalarfij@kfu.edu.sa (A.A. Alarfaj).
} 


\section{Literature review}

Mukhopadhyay C. et al. ${ }^{8}$ in 2008 had conducted an experimental study. 50 samples were collected and divided into two groups according to the frequency of using earbuds and the samples were processed. In group A, bacteria were found in 20 (80\%) ear and 14 (56\%) earbuds swabs. In group B, bacteria were found in 23 (92\%) ear and 17 (68\%) earbuds swabs. Group B showed increased bacterial growth due to frequent and constant use. It was concluded that frequent and constant use of earbuds encourages bacterial growth in the external ear canal and sharing of earbuds might be a potential vector of commensals. Therefore, it is advised to not share earbuds or to clean them before sharing. ${ }^{8}$

A questionnaire based-study was published in March 2019 by Zia, S. et al. ${ }^{9}$ It was an observational study conducted at the Outpatient Department of Dow University Hospital, OJHA campus, Karachi. It was carried out for one year starting on December 2016 until December 2017. Patients attending outpatient department filled out the questionnaire. Information regarding the variables of earbuds, cotton bud use and swimming along with ear infection and side of infection, recent history of respiratory tract infection/trauma to ear canal, ear surgery and symptoms like pain, bleeding, itching, fever, hearing loss, pus and whistling in ear were collected. ${ }^{9}$ They found out that ear pain was observed in majority individuals followed by itching, whistling in ear, fullness in ear, fever, pus, hearing loss, while bleeding was observed in 19 individuals. The conclusion drown out from the results was that there was an association between cotton bud and headphone use with Otitis Externa. $^{9}$

\section{Methods}

A cross sectional questionnaire-based study was conducted after an electronic questionnaire validated by experts was shared online within the targeted population. As this study did not have any intervention, an ethical approval was not required. The survey was pretested with a small sample (20 participants) in order to receive feedback and correct any confusion. For a questionnaire with $95 \%$ confidence interval and $5 \%$ acceptable error margin, we needed at least 407 participants based on the area's population calculated with the Survey System calculator. In this study, 504 responses have been collected.

Inclusion criteria: participants who were from Alahssa, Dammam, Qatif, or Jubail industrial city, use earbuds, older than 12 years of age and gave consent.

Exclusion criteria: teenagers younger than age 12 years, previously diagnosed hearing loss, existing acute or chromic ear disease, current hearing aid users.

The questionnaire consisted of 19 elements including a consent, 6 questions for sociodemographic data, 6 questions for the attitude of earbuds use, 2 for assessment of side effects, and 4 questions to assess the sample's awareness. The total score of awareness was obtained by adding up the 4 questions. We further categorized 0 to 1 as poor awareness, 2 was classified as moderate and 3 to 4 as good awareness. Data was stored in a soft copy-Excel sheet, and the survey was anonymous.

\section{Statistical method}

Descriptive Statistics were illustrated with counts, proportions (\%), mean \pm standard deviation as appropriate. Between-group comparisons are achieved with Mann Whitney $U$ test ( 2 categories) and Kruskal Wallis Test (3 or more categories) for mixed variables, whenever appropriate. All statistical tests are two-sided and $p$ value $<0.05$ is considered statistically significant. Normally test has been conducted using Kolmogorov-Smirnov and Shapiro-Wilk test, $\mathrm{p}<0.05$ were considered skewed data. The whole data analyses had been accomplished with Statistical Packages for Software Sciences (SPSS) version 21 (SPSS, Armonk, NY: IBM Corp., USA).

\section{Results}

We recruited 504 participants including adolescents and adults among 4 cities; AlAhssa (45\%), Al Jubail Industrial City (40.1\%), Dammam (9.1\%) and AlQatif (5.8\%). As seen in Table 1, the age range was between 12 and 65 years old, with the dominance of age range between 21 and 30 years (43.7\%). Female and male ratio was 3.1:1. Unmarried participants percentage was slightly higher (57.5\%) compared to the percentage married participants (42.5\%). Majority of the participants were students (55\%) with approximately three quarters of them in the educational level of bachelor degree.

Table 2 presented the behavior of participants toward the use of earbuds. Based on the results, $46.6 \%$ of them were using earbuds for 1-5 years, with many of them were using it daily $(32.5 \%)$ for about $1-2 \mathrm{~h}$ (49.8\%). When asked if they fall asleep while listening to a playing sound using earbuds, only $18.1 \%$ stated that they were using earbuds even when sleeping. When asked about the level of earbuds volume, majority of them reported intermediate volume (68.7\%). Furthermore, there were $20 \%$ who had experienced symptoms due to the long-term usage of earbuds and the most common of them were decreased hearing $(10.1 \%)$ and infection of inflammation of the external ear canal (7.3\%).

Fig. 1 described the symptoms that were experienced by the participants while using earbuds and the most common of them was itching (28\%), followed by otalgia or pain in the ear (23\%) and excessive ear wax $(21.8 \%)$ while the least of them was Tinnitus or ringing in the ear (16.5\%).

In the assessment of awareness toward the side effect of earbuds use, we observed that approximately three-fourth of them (74.8\%) would not exchange earbuds with others, while the awareness of cleaning earbuds regularly was poor $(29.8 \%)$. Furthermore, more than a half of them (56.3\%) had previous knowledge regarding the side effect and long-term complication of prolonged use of earbuds and after learning this, nearly all (86.9\%) had decided to decrease the hours of earbuds use and they clean the earbuds regularly. The overall mean awareness score showed 2.49 (SD 0.95) (Table 3) (see Table 4).

Fig. 2 depicted the level of awareness toward the side effect of earbuds use. It was revealed that a little below a half of them were classified as good awareness, $35.3 \%$ were of moderate awareness and the

Table 1

Description of socio-demographic characteristics $(n=504)$

\begin{tabular}{ll}
\hline Study Variables & $\mathrm{N}(\%)$ \\
\hline Age group & \\
- $12-20$ years & $128(25.4 \%)$ \\
- $21-30$ years & $220(43.7 \%)$ \\
- $31-40$ years & $91(18.1 \%)$ \\
- >40 years & $65(12.9 \%)$ \\
Gender & \\
- Male & $122(24.2 \%)$ \\
- Female & $382(75.8 \%)$ \\
Marital status & \\
- Unmarried & $290(57.5 \%)$ \\
- Married & $214(42.5 \%)$ \\
Residence city & \\
- Al Ahsa & $227(45.0 \%)$ \\
- Al Jubail Industrial City & $202(40.1 \%)$ \\
- Dammam & $46(09.1 \%)$ \\
- Qatif & $29(05.8 \%)$ \\
Employment status & \\
- Employed & $141(28.0 \%)$ \\
- Unemployed & $86(17.1 \%)$ \\
- Student & $277(55.0 \%)$ \\
Educational level & \\
- Primary school & \\
- Intermediate school & \\
- High school & \\
- Bachelor degree & \\
- Postgraduate & $26(05.80 \%)$ \\
\hline
\end{tabular}


Table 2

Participants attitude toward the use of earbuds $(n=504)$.

\begin{tabular}{|c|c|}
\hline Statement & $\mathrm{N}(\%)$ \\
\hline \multicolumn{2}{|l|}{ Years of using Earbuds } \\
\hline - $1-5$ years & $235(46.6 \%)$ \\
\hline - 6-10 years & $209(41.5 \%)$ \\
\hline - $>10$ years & $60(11.9 \%)$ \\
\hline \multicolumn{2}{|l|}{ Number of days per week in using Earbuds } \\
\hline - One day & $87(17.3 \%)$ \\
\hline - Two days & $52(10.3 \%)$ \\
\hline - Three days & $60(11.9 \%)$ \\
\hline - Four days & $58(11.5 \%)$ \\
\hline - Five days & $59(11.7 \%)$ \\
\hline - Six days & $24(04.8 \%)$ \\
\hline - Seven days & $164(32.5 \%)$ \\
\hline \multicolumn{2}{|l|}{ Hours per day in using Ear buds } \\
\hline - $1-2 \mathrm{~h}$ & $251(49.8 \%)$ \\
\hline - $3-4 \mathrm{~h}$ & $121(24.0 \%)$ \\
\hline - 5-6 h & $56(11.1 \%)$ \\
\hline - $7-8 \mathrm{~h}$ & $30(06.0 \%)$ \\
\hline - $9-10 \mathrm{~h}$ & $22(04.4 \%)$ \\
\hline - $11-12 \mathrm{~h}$ & $08(01.6 \%)$ \\
\hline • $13-14 \mathrm{~h}$ & $16(03.2 \%)$ \\
\hline \multicolumn{2}{|l|}{ Fall asleep while listening to a playing sound via earbuds } \\
\hline - Yes & $91(18.1 \%)$ \\
\hline - No & $413(81.9 \%)$ \\
\hline \multicolumn{2}{|l|}{ Level of the Earbuds volume } \\
\hline - Low & $74(14.7 \%)$ \\
\hline - Intermediate & $346(68.7 \%)$ \\
\hline - High & $84(16.7 \%)$ \\
\hline \multicolumn{2}{|c|}{ Experience any of these symptoms due to a long-term use of earbuds } \\
\hline - Decrease hearing & $51(10.1 \%)$ \\
\hline - Infection or inflammation of the external ear canal & $37(07.3 \%)$ \\
\hline - None of the above & $403(80.0 \%)$ \\
\hline - Other & $13(02.6 \%)$ \\
\hline
\end{tabular}

remaining were poor (15.5\%).

In Fig. 3, the prevalence of regular (daily) use of earbuds was $32.5 \%$ while those who were using irregularly was $67.5 \%$ ).

When measuring the association between awareness among the socio-demographic characteristics and the behavior of participants toward the use of earbuds, it was found that, the awareness of females was significantly better compared to males $(\mathrm{T}=-2.569 ; \mathrm{P}=0.010)$. We also have known that the awareness of students was significantly less when compared to the other group $(\mathrm{F}=2.824 ; \mathrm{P}=0.044)$. In addition, the awareness of those participants who were using earbuds for ${ }^{1,2}$ hours was significantly higher when compared to those who were using for more than $2 \mathrm{~h}((\mathrm{~F}=2.901 ; \mathrm{P}=0.040)$ whereas the awareness of those participants who were using a high level of earbuds volume was significantly poor when compared to other group ( $\mathrm{F}=9.617 ; \mathrm{P}=<0.001)$. On the other hand, age group, marital status, residence city, educational level, years of using earbuds, number of days per week in using earbuds, falling asleep while using earbuds and experience symptoms due to long-term use of earbuds were not statistically significant when compared to the awareness toward the side effect of earbuds use.

Fig. 4 shows that the majority of participants (92.7\%) use earbuds, while only $7.3 \%$ (that is 37 participants) use headphones.

\section{Discussion}

The purpose of this study is to determine the awareness of teenagers and adults regarding the side effect of earbuds use. The awareness of the respondents in this study was good among $49.2 \%$ while moderate and poor awareness were found among 35.3 and $15.5 \%$ of respondents, respectively. The mean awareness score was 2.49 (SD 0.95) out of 4 points. Furthermore, females showed significantly better awareness when compared to males $(\mathrm{T}=-2.569 ; \mathrm{P}=0.010)$ while those who were using earbuds for $1-2 \mathrm{~h}$ were more associated with higher awareness level as opposed to the other groups $(\mathrm{F}=2.901 ; \mathrm{P}=0.040)$. On the other hand, students $(\mathrm{F}=2.824 ; \mathrm{P}=0.044)$ and those who were using high level of volume ( $\mathrm{F}=9.617 ; \mathrm{P}=<0.001$ ) were significantly more of having poor level of awareness. To our knowledge, this is the first paper in Saudi Arabia that measured the awareness level and its associated factors in regards to the side effect due to the continuous usage of earbuds among the general population, which was an important finding given the use of earbuds at the present time is widely prevalent among teenagers and young adults. Moreover, since the idea is novel, we hope that our results had shed more light in the growing investigations to the relative perception to earbuds use and its side effect.

Frequent use of earbuds would cause complication to ear. In our study, the regular use of earbuds had been detected to $32.5 \%$ of the respondents. This result did not seem to disagree with the paper of Pandey et al., ${ }^{10}$ It was an observational cross-sectional study that measured the prevalence of headphone usage among medical

Table 3

Awareness of Participants Toward Earbuds side effect ${ }^{(n=504)}$.

\begin{tabular}{|c|c|}
\hline Statement & $\begin{array}{l}\text { Correct } \\
\text { Answer } \\
\mathrm{N}(\%)\end{array}$ \\
\hline Do you exchange earbuds with others? ${ }^{a}$ & $377(74.8 \%)$ \\
\hline Do you clean earbuds regularly? & $150(29.8 \%)$ \\
\hline $\begin{array}{l}\text { Do you have a former knowledge of the side effects and the long- } \\
\text { term complications of prolonged use of earbuds? }\end{array}$ & $284(56.3 \%)$ \\
\hline $\begin{array}{l}\text { After learning about the side effects and the complications due to a } \\
\text { prolonged use of earbuds, are you going to apply the healthier } \\
\text { attitude by decreasing the number of hours of use and regularly } \\
\text { cleaning the earbuds? }\end{array}$ & $438(86.9 \%)$ \\
\hline Total Awareness Score & $2.49 \pm 0.95$ \\
\hline
\end{tabular}

${ }^{\mathrm{a}}$ Indicates reverse answer.

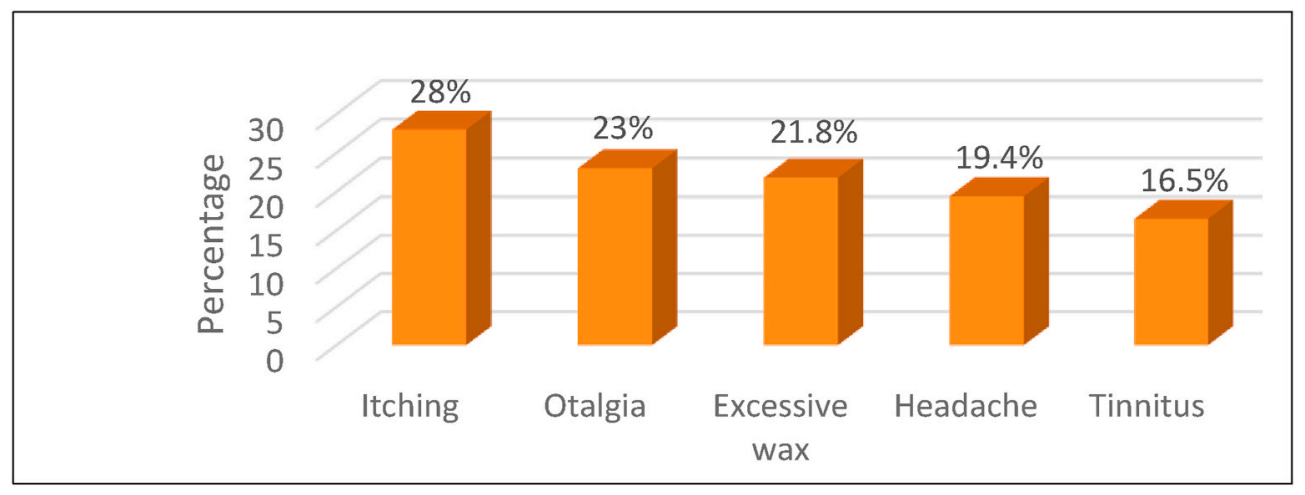

Fig. 1. Direct symptoms after using earbuds. 
Table 4

Statistical association to determine the factors associated with the awareness toward the side of earbuds use ${ }^{(n=504)}$.

\begin{tabular}{|c|c|c|c|}
\hline Factor & $\begin{array}{l}\text { Awareness } \\
\text { Total Score (4) } \\
\text { Mean } \pm \text { SD }\end{array}$ & $\mathrm{F} / \mathrm{T}$ test & P-value \\
\hline \multicolumn{4}{|l|}{ Age group ${ }^{a}$} \\
\hline - 12-20 years & $2.37 \pm 0.96$ & $\mathrm{~F}=1.640$ & 0.158 \\
\hline - 21-30 years & $2.45 \pm 0.99$ & & \\
\hline - $31-40$ years & $2.58 \pm 0.84$ & & \\
\hline \multicolumn{4}{|l|}{ Gender $^{\mathrm{b}}$} \\
\hline - Male & $2.29 \pm 0.95$ & $\mathrm{~T}=-2.569$ & $0.010 * *$ \\
\hline \multicolumn{4}{|l|}{ Marital status ${ }^{\mathrm{b}}$} \\
\hline - Unmarried & $2.41 \pm 0.99$ & $\mathrm{~T}=-1.871$ & 0.068 \\
\hline \multicolumn{4}{|l|}{ Residence city ${ }^{\mathrm{a}}$} \\
\hline - Al Ahsa & $2.41 \pm 0.89$ & $\mathrm{~F}=1.279$ & 0.271 \\
\hline - Al Jubail Industrial City & $2.49 \pm 1.02$ & & \\
\hline - Dammam & $2.69 \pm 0.92$ & & \\
\hline - Qatif & $2.59 \pm 0.87$ & & \\
\hline \multicolumn{4}{|l|}{ Employment status $^{\mathrm{a}}$} \\
\hline - Employed & $2.53 \pm 0.88$ & $\mathrm{~F}=2.824$ & $0.044 * *$ \\
\hline - Unemployed & $2.66 \pm 0.94$ & & \\
\hline - Student & $2.39 \pm 0.98$ & & \\
\hline \multicolumn{4}{|l|}{ Educational level $^{\mathrm{b}}$} \\
\hline - High school or below & $2.49 \pm 0.95$ & $\mathrm{~T}=0.170$ & 0.834 \\
\hline \multicolumn{4}{|l|}{ Years of using Earbuds ${ }^{\mathrm{a}}$} \\
\hline - 1-5 years & $2.50 \pm 0.06$ & $\mathrm{~F}=1.437$ & 0.242 \\
\hline - 6-10 years & $2.51 \pm 0.98$ & & \\
\hline - $>10$ years & $2.28 \pm 0.94$ & & \\
\hline \multicolumn{4}{|l|}{ Regular (daily) use of earbuds ${ }^{\mathrm{b}}$} \\
\hline - Yes & $2.42 \pm 1.02$ & $\mathrm{~T}=-0.943$ & 0.266 \\
\hline - No & $2.51 \pm 0.91$ & & \\
\hline \multicolumn{4}{|l|}{ Hours per day in using Ear buds ${ }^{\mathrm{a}}$} \\
\hline - $1-2 \mathrm{~h}$ & $2.58 \pm 0.89$ & $\mathrm{~F}=2.901$ & $0.040 * *$ \\
\hline - $3-4 \mathrm{~h}$ & $2.35 \pm 0.95$ & & \\
\hline - $\geq 5 \mathrm{~h}$ & $2.41 \pm 1.04$ & & \\
\hline \multicolumn{4}{|c|}{ Fall asleep while listening to a playing sound via earbuds ${ }^{\mathrm{b}}$} \\
\hline - Yes & $2.38 \pm 1.05$ & $\mathrm{~T}=-1.038$ & 0.353 \\
\hline \multicolumn{4}{|l|}{ Level of the Earbuds volume ${ }^{a}$} \\
\hline - Low & $2.91 \pm 0.94$ & $\mathrm{~F}=9.617$ & $<0.001 * *$ \\
\hline - Intermediate & $2.43 \pm 0.91$ & & \\
\hline - High & $2.31 \pm 1.04$ & & \\
\hline \multicolumn{4}{|c|}{ Experience symptoms due to a long-term use of earbuds ${ }^{b}$} \\
\hline - Yes & $2.53 \pm 0.90$ & $\mathrm{~T}=0.668$ & 0.499 \\
\hline - No & $2.46 \pm 0.96$ & & \\
\hline
\end{tabular}

** Significant at $\mathrm{p}<0.05$ level.

a P-value has been calculated using Kruskal Wallis test.

b P-value has been calculated using Mann Whitney $U$ test.

undergraduates while driving. They found out that the prevalence of regular usage of headphone while driving was $20.8 \%$. On the other hand, the prevalence with regards to the regular usage of personal listening devices during studying of Rekha et al., ${ }^{11}$ was relatively higher
(83.1\%). This result has been corroborated by Sunny et al., ${ }^{12}$ which reported a prevalence rate of earbuds use among the students and subjective tinnitus was $95.6 \%$ which was far off distant that the prevalence of regular usage of earbuds in our study. Moreover, approximately half of the respondents in our study had a duration of earbuds usage for $1-2 \mathrm{~h}$ per day. Which was lower than that in a paper of Awad et al., ${ }^{13}$ where they indicated $4 \mathrm{~h}$ or more of use per day of headphone. Another paper published in India by Sachdeva and Kumar, ${ }^{14}$ reported that many of the students were using headphones in a duration of 2-4 h (35\%) or 4-6 h (27\%) per day which was higher than the finding in our report. On the other hand, majority of students (77.7\%) in a paper of Sunny et al., ${ }^{12}$ indicated $1 \mathrm{~h}$ of use of earphone per day which was in line with our study findings.

In this study, the most commonly known symptoms toward the longterm use of earbuds were itching (28\%), followed by otalgia -or pain in the ear- $(23 \%)$ then excessive ear wax $(21.8 \%)$. These findings may coincide with the findings of the paper of Mohammadpoorasl et al., ${ }^{15}$ They documented that the most frequently mentioned ear complication due to the excessive usage of headphones were ear disease, hearing loss, ear infection, ringing in the ears, and dizziness. Similarly, in Zia, S. et al., ${ }^{16}$ they accounted Tinnitus or ringing in the ear (20\%), followed by hearing difficulty and numbness (both 14\%, respectively). However, Rekha et al., ${ }^{11}$ provided conflicting reports with regards to the symptoms associated with the continuous usage of personal listening device. They observed that the most common complications were hard of hearing feeling, waking, suffering from lack of sleep and wheezing or buzzing.

Moreover, in our study, nearly three-fourth of the respondents never shared their earbuds to others with only $24.2 \%$ agreed to share it. However, the awareness of cleaning the earbuds regularly was poor (29.8\%). We also observed that nearly all agreed (86.9\%) that decreasing the number of hours in using earbuds was necessary to avoid ear complication. The attitude of sharing earphones to others was higher in the paper of Sachdeva and Kumar, ${ }^{14}$ They documented that nearly half of the students (49\%) had shared their earphones with others while the habit of cleaning the earphone regularly was higher compared to the finding of our study which was $49 \%$ compared to our study with only $29.8 \%$.

\section{Conclusion}

There was an adequate awareness toward the side effect of excessive usage of earbuds among teenagers and young adults. Females showed better awareness compared to males and shorter duration of usage of earbuds was a significant predictor of good awareness. On the other hand, students showed poor awareness compared to the other groups while playing sound while wearing earbuds in a high volume was the significant predictor of poor awareness. Further research is needed in order to validate the good level of awareness among the general population and its relationship to the long-term effect of excessive usage of earbuds in our region.

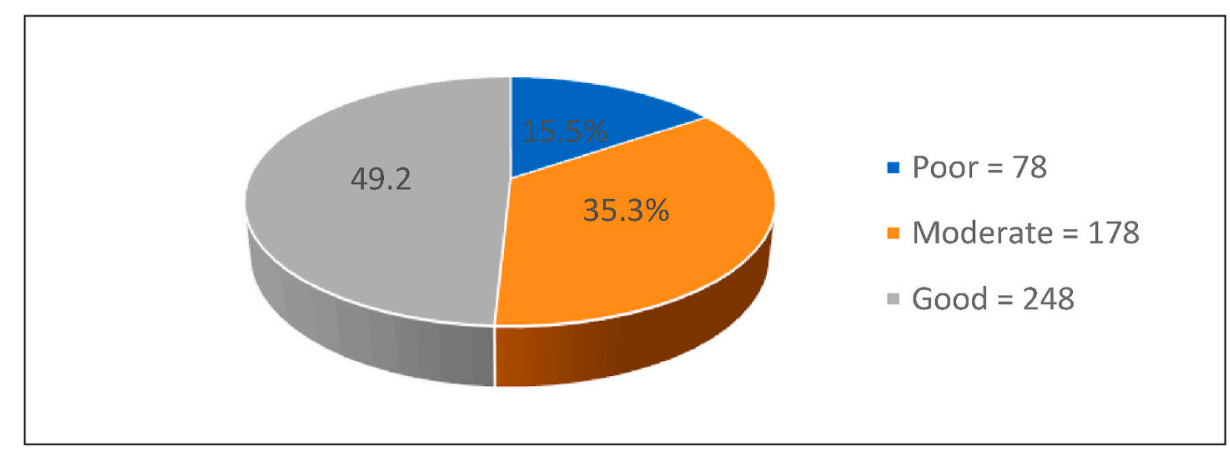

Fig. 2. Level of Awareness toward Earbuds side effect. 


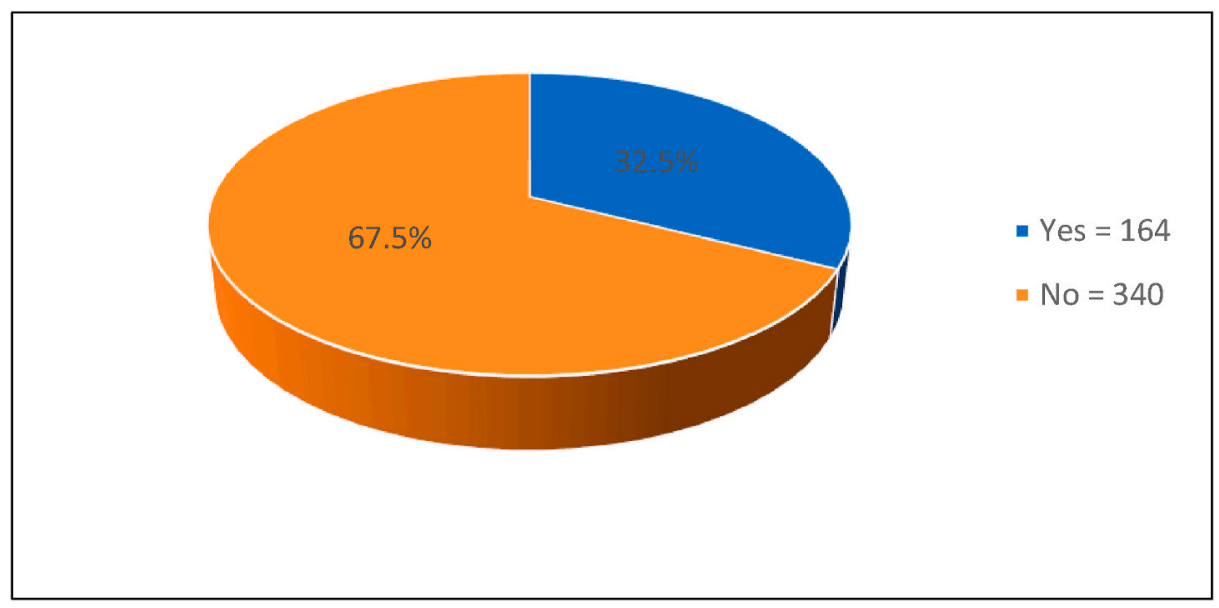

Fig. 3. Prevalence of regular usage of earbuds.

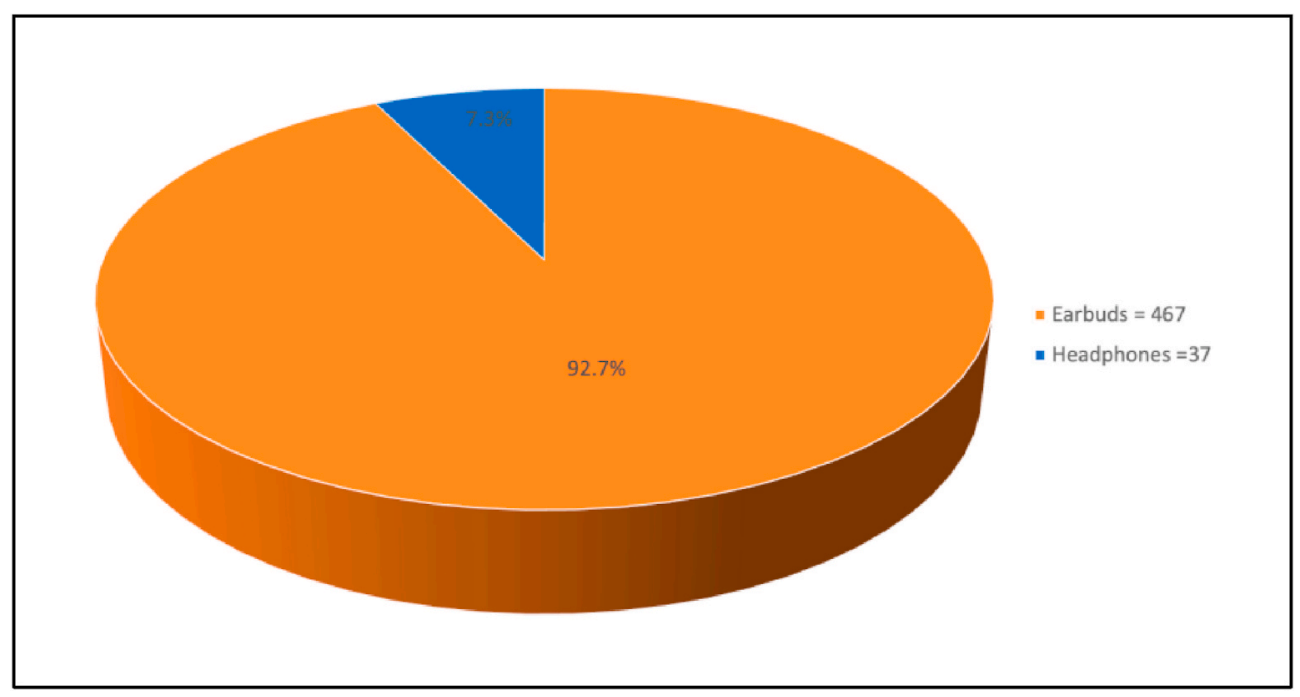

Fig. 4. Prevalence of earbuds use instead of headphones among participants.

\section{Recommendations for further research}

Further research is needed in order to validate the good level of awareness among the general population and its relationship to the longterm effect of excessive usage of earbuds in our region. Also, a laboratory-based research is recommended to compare the bacterial growth of the external ear canal in association with earbuds use in different frequencies and assess the role of earbuds as vector of bacteria.

\section{Acknowledgment}

The great deal of support provided by the College of Medicine, King Faisal University, AlAhssa, Saudi Arabia is sincerely acknowledged. In addition, we acknowledge the cooperation and enlightenment extended by Dr. Ebtisam Ahmed, an expert of Public health, KFU.

\section{References}

1 Silbergleid, M. and Mark Schubin, S., n.d. Blogs | Sports Video Group. [online] Sports Video Group. Available at: <https://www.sportsvideo.org/blogs/?blog=schu bin-cafe/ $>$ [Accessed 17 March 2020].

2 Newman, M., 2017. The History Of Headphones. [online] Cool Material. Available at: <https://coolmaterial.com/roundup/history-of-headphones/> [Accessed 17 March 2020].

3 Singer M. Nathaniel Baldwin, Utah Inventor and Patron of the Fundamentalist Movement [ebook]. first ed. Utah: Department of Anthropology, University of Utah; 1929:43.
Available at: https://collections.lib.utah.edu/dl_files/78/49/78499517b0bfdbe5c97 d7e639888f8d1083597a4.pdf. Accessed March 18, 2020.

4 Forums.hardwarezone.com.sg. Historic Headphones [online] Available at:; 2012. https://forums.hardwarezone.com.sg/headphones-earphones-portable-media-devic es-314/historic-headphones-3765907.html. Accessed March 16, 2020.

5 Sony.com. Sony Corporation of America Press Releases [online] Available at:; 2019. htt ps://www.sony.com/content/sony/en/en_us/SCA/company-news/press-releases. sony-electronics.2019.html. Accessed March 17, 2020.

6 Global Sony. Sony Design- History-1980s [ONLINE] Available at:; 2020. https://www. sony.net/Fun/design/history/product/1980/mdr-w30l.html. Accessed March 17, 2020.

7 Earphones Customised. Headphone and Earphone History [ONLINE] Available at:; 2014. http://www.ear-phones.co.za/headphone-ear-phone-history/. Accessed March 18, 2020.

8 Mukhopadhyay C. A comparative analysis of bacterial growth with earphone use. Online J Health Allied Sci. 2008;7(2):1-3. Apr-Jun 2008.

9 Zia S, Tahir H, Azeem K, Adil SO, shehzad A, shah M. Frequency and factors OF ear infection among swimmers, cotton bud and headphone users. Pakistan J. Public Health. 2019;9(1):15-18. https://doi.org/10.32413/pjph.v9i1.223.

10 Pandey D, Saroshe S, Dixit S, Sabde Y. Estimation of prevalence of headphone usage during driving and awareness about their health hazards among medical undergraduates. Int. J. Community Med. Public Health. 2015 Apr;2(2):167.

11 Rekha T, Unnikrishnan B, Mithra PP, Kumar N, Bukelo MJ, Ballala K. Perceptions and practices regarding use of personal listening devices among medical students in coastal South India. Noise Health. 2011 Sep 1;13(54):329.

12 Sunny OD, Asoegwu CN, Abayomi SO. Subjective tinnitus and its association with use of ear phones among students of the College of Medicine, University of Lagos, Nigeria. Int Tinnitus J. 2012 Dec 19;17(2):169-172.

13 Awad M, Ali S, Ayed S, et al. Impact of headphone use on hearing among medical students at king Khalid University, Saudi Arabia. Med J Cairo Univ. 2016;84(2): 93-97. 
14 Sachdeva S, Kumar M. Study on health impacts of ear and headphones among students lives in Chandigarh. Int J Res Appl Sci Eng Technol. 2018;6(3).

15 Mohammadpoorasl A, Hajizadeh M, Marin S, Heydari P, Ghalenoei M. Prevalence and pattern of using headphones and its relationship with hearing loss among students. Health Scope. 2018 Jan 1;7(4).
16 Zia S, Tahir HM, Azeem K, Adil SO. Frequency and factors of ear infection among swimmers, cotton bud and headphone users. Pakistan J. Public Health. 2019 Jul 13;9 (1):15-18. 